\title{
A NOTE ON MODELS OF IDENTITIES
}

\author{
A. K. AUSTIN
}

The object of this note is to shed light on the question, "Is there an algorithm for determining whether an identity implies the identity $x=y$ ?" The example in this paper shows that testing finite models will not be an algorithm.

S. K. Stein shows in [1] that there exists a set of identities which has infinite models but no finite models other than the model with only one element. He proves the result for an infinite set of identities; in this note the result will be proved for a set containing only one identity.

THEOREM. The identity,

$$
((y y \cdot y) x)((y y \cdot(y y \cdot y)) z)=x,
$$

has infinite models but no finite models other than the model with only one element.

The proof of the theorem consists of the following two lemmas.

LEMMA 1. The identity (1) has an infinite model.

Proof. The model is defined on the set of positive integers as follows:

$$
\begin{aligned}
& y y=4 y+1,(4 y+1) \cdot y=4 y+3,(4 y+3) \cdot x=(4 x+2) \text { if } x \neq 4 y+3, \\
& (4 y+1) \cdot(4 y+3)=4 y,(4 y) \cdot z=4 y \text { if } z \neq 4 y,(4 x+2) \cdot(4 y)=x, \\
& (4 x+2) \cdot(4 z+1)=x,(16 y+13) \cdot(4 y)=4 y+3, \\
& (16 y+13) \cdot(16 y+1)=4 y+3, x y=1 \text { otherwise. }
\end{aligned}
$$

LEMma 2. Any finite model of (1) contains only one element.

Proof. Let $G$ be a finite model of (1) and let $q(y)=(y y)(y y \cdot y)$ and $t(y)=y y \cdot y$. Let $R_{u}$ and $L_{u}$ denote right and left translation by $u$. Then (1) can be written

$$
(t(y) \cdot x)(q(y) \cdot z)=x,
$$

or

$$
R_{q(y) \cdot z} L_{t(y)}=I
$$

the identity map of $G$.

Received by the editors February 12, 1964. 
From (3), $R_{q(y) \cdot z}$ is onto and $L_{t(y)}$ is $1-1$. Since $G$ is finite, both are bijections. In particular, letting $a$ be any element of $G$, there is an element $b$ such that $t(a) \cdot b=t(a)$. Thus $b=((t(a)) b)(q(a) \cdot z)$ $=(t(a))(q(a) \cdot z)$. Since $L_{t(a)}$ is $1-1, q(a) \cdot z$ is independent of $z$. Thus $L_{q(a)}$ is constant.

Let $c=q(a) \cdot z$, independent of $z$. Replacing $z$ by $q(y) \cdot z$ gives $c=q(a)(q(y) \cdot z)$. From (2), with $x$ replaced by $c$,

$$
c=(t(y) \cdot c)(q(y) \cdot z) \text {. }
$$

Since $R_{q(y) \cdot z}$ is $1-1, t(y) \cdot c=q(a)$, which is independent of $y$. Thus $t(y) \cdot c=t(a) \cdot c$ and since $R_{c}$ is $1-1, t(y)=t(a)$.

Now let $y$ be replaced by $d=t(a)$. This gives $t(d)=t(a)=d$. Thus $q(d)=d d \cdot t(d)=d d \cdot d=t(d)$.

Since $q(d)=t(d), L_{q(d)}$, which is constant, is equal to $L_{t(d)}$, which is a bijection. Thus $G$ has only one element.

\section{REFERENCE}

1. S. K. Stein, Finite models of identities, Proc. Amer. Math. Soc. 14 (1963), 216-222.

Training College, Huld, Yorkshire, England

\section{AN ELEMENTARY INTERPOLATION THEOREM}

\section{DAVID G. CANTOR AND R. R. PHELPS}

In this note we prove a simple lemma concerning interpolation properties of certain families of maps in semigroups. The main application is to the family of finite Blaschke products, considered as maps of the circle into itself. (Other applications are possible, but most of them are well known.)

Consider a semigroup $S$ and a family $F$ of maps from $S$ into $S$. We will call $F n$-transitive if for any $n$ distinct elements $\alpha_{1}, \alpha_{2}, \cdots, \alpha_{n}$ of $S$ and arbitrary elements $\beta_{1}, \beta_{2}, \cdots, \beta_{n}$ in $S$, there exists $f$ in $F$ such that $f\left(\alpha_{i}\right)=\beta_{i}, i=1,2, \cdots, n$.

Lemma. Suppose that $S$ is a semigroup with (two-sided) identity 1 and suppose there is an element $\beta$ in $S$ with $\beta \neq \beta^{2} \neq 1$. Assume, furthermore, that $F$ is a family of maps (from $S$ into $S$ ) which is closed under

Received by the editors December 17, 1963. 